\title{
Inverse statistics in stock markets: Universality and idiosyncracy
}

\author{
Wei-Xing Zhou ${ }^{\text {a,1 }}$, Wei-Kang Yuan ${ }^{\text {a }}$ \\ a State Key Laboratory of Chemical Reaction Engineering, \\ East China University of Science and Technology, Shanghai 200237, China
}

\begin{abstract}
Investigations of inverse statistics (a concept borrowed from turbulence) in stock markets, exemplified with filtered Dow Jones Industrial Average, S\&P 500, and NASDAQ, have uncovered a novel stylized fact that the distribution of exit time follows a power law $p\left(\tau_{\rho}\right) \sim \tau_{\rho}^{-\alpha}$ with $\alpha \approx 1.5$ at large $\tau_{\rho}$ and the optimal investment horizon $\tau_{\rho}^{*}$ scales as $\rho^{\gamma}[1,2,3]$. We have performed an extensive analysis based on unfiltered daily indices and stock prices and high-frequency (5-min) records as well in the markets all over the world. Our analysis confirms that the power-law distribution of the exit time with an exponent of about $\alpha=1.5$ is universal for all the data sets analyzed. In addition, all data sets show that the power-law scaling in the optimal investment horizon holds, but with idiosyncratic exponent. Specifically, $\gamma \approx 1.5$ for the daily data in most of the developed stock markets and the five-minute highfrequency data, while the $\gamma$ values of the daily indexes and stock prices in emerging markets are significantly less than 1.5. We show that there is of little chance that this discrepancy in $\gamma$ stems from the difference of record sizes in the two kinds of stock markets.
\end{abstract}

Key words: Econophysics; Stock markets; Stylized fact; Inverse statistics; Exit time; Power law

PACS: 89.65.Gh, 02.50.-r, 89.90.+n

\section{Introduction}

Econophysics is an interdisciplinary science which applies statistical physics and complex system theories to economics [4,5,6,7]. More than ten stylized

1 Corresponding author. 130 Meilong Road, East China University of Science and Technology, P.O. Box 369, Shanghai 200237, China

E-mail address: wxzhou@moho.ess.ucla.edu (W.-X. Zhou)

Preprint submitted to Elsevier Science 22 September 2018 
facts of asset returns have been discovered or re-discovered in the community [8], some of which are inspired originally by the analogy between finance markets and turbulence $[9,10]$. In these works, the asset return, as the counterpart of velocity difference in turbulence, plays a central role, which is defined as the difference of logarithmic prices at a given time lag. Recently, a new stylized fact have been unveiled dealing with the inverse statistics of the exit time in the Dow Jones Industrial Average $[1,2,3]$ and in the foreign exchange markets [11]. Interestingly, this concept of inverse statistics was also borrowed from turbulence [12] and applied in turbulence extensively [13,14,15,16,17,18].

For a given series of $\log$ prices $\left\{s_{i}\right\}$ where $i$ corresponds to trading days, the exit time (or first passage time) $\tau$ at time $i$ for a given return threshold $\rho>0$ is defined as the minimal time span needed for the difference of log prices exceeds $\rho$ for the first time. In other words, one says mathematically

$$
\tau_{\rho}=\inf \left\{j-i: s_{j}-s_{i} \geq \rho, j>i\right\} .
$$

We see that $\tau_{\rho} \geq 1$ is integer. If the stock price rises, $\tau_{\rho}=1$. It is argued that more small $\tau_{\rho}$ in a period implies a bullish market while large $\tau_{\rho}$ indicates a lasting bearish market. For fractional Brownian motion of Hurst exponent $H$, Ding and Yang [19] have found that the distribution density $p\left(\tau_{\rho}\right)$ scales as

$$
p\left(\tau_{\rho}\right) \sim \tau_{\rho}^{-\alpha}
$$

with $\alpha=2-H$, when $\tau_{\rho}$ is large. For Brownian motion, the exit time distribution has been solved analytically [20,21,22]

$$
p\left(\tau_{\rho}\right)=\frac{\rho}{\sqrt{4 \pi K \tau_{\rho}^{3}}} \exp \left[-\rho^{2} / 4 K \tau_{\rho}\right],
$$

where $K$ is the generalized diffusion constant. The most probable exit time $\tau_{\rho}^{*}$ (also called the optimal investment horizon in Finance) is thus scaled as

$$
\tau_{\rho}^{*}=2 \rho^{2} / 3
$$

The first passage time was studied in physics, biology and engineering $[20,21,22$, and references therein]. When applying to financial markets, the probability distributions of the exit time of the filtered DJIA follow a power law as expressed by Eq. 2 with $\alpha=1.5[1,2,3]$ and the foreign exchange rates show $\alpha=2.4$ [11]. For the filtered DJIA, S\&P 500 and NASDAQ, the optimal investment horizon scales against $\rho$ as

$$
\tau_{\rho}^{*} \sim \rho^{\gamma}
$$

with $\gamma=1.8$, remarkably different from $2[1]$. 
In this paper, we shall perform an extensive investigation of the inverse statistics in the stock markets. The data sets consist of daily stock indexes and stock prices from different markets and high-frequency (five minutes) data as well. We don't filter the data so that it is more rational to refer to $\rho$ as the return threshold.

\section{Probability distribution of exit time}

The probability distribution of the DJIA has been studied and the scaling exponent is determined to be $\alpha=1.5[1,2]$. To further explore the inverse statistics of the DJIA, we investigated the daily prices of 37 stocks that are or were components of DJIA since 1962. The empirical probability densities $p\left(\tau_{\rho}\right)$ were obtained with the aim of Gaussian kernel smoothing estimation [23]. The empirical probability distributions of the exit time for all 37 stocks at three different levels $\rho=0.00954,0.0596$, and 0.244 are shown in Fig. 1 with different line types. For a given $\rho$, the DJIA components have similar shapes in their probability distributions. This figure also shows the evolution of the distribution with respect to $\rho$. The power-law scaling is clearly visible for large $\tau_{\rho}$. We find that the exponent of the power law is also $\alpha \approx 1.5$, in agreement with that of the DJIA.

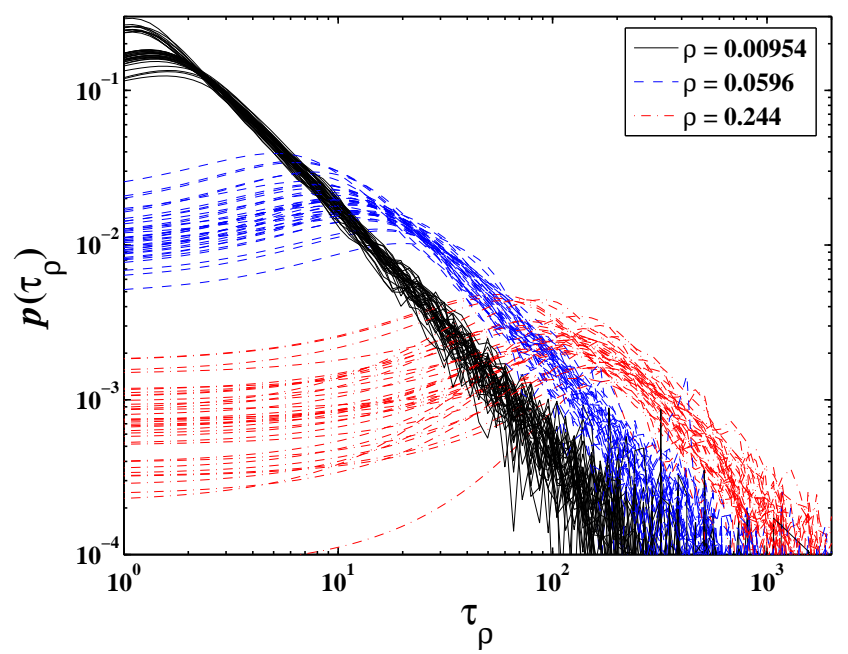

Fig. 1. The empirical probability distributions of the exit time of daily prices of 37 stocks that are or were components of DJIA since 1962 at three different levels $\rho=0.00954,0.0596$, and 0.244 .

We also performed analysis on the daily evolution of the S\&P 500 index (from $1940 / 11 / 29$ to $2004 / 09 / 21$, totally 16115 data points) and of the NASDAQ index (from 1971/02/05 to 2002/11/15, totally 8025 data points) in the USA stock markets. The power-law scaling distribution of the exit time with an exponent of about 1.5 is confirmed for both indexes, as reported in $[2,3]$. 
A question arises naturally asking whether this new statistical property holds in other stock markets other than the USA. To address this question, we have carried out the same analysis on the following 40 indexes all over the world: Argentina (from 1996/10/08 to 2004/09/20), Australia (from 1984/08/03 to 2004/09/21), Austria (from 1986/01/08 to 2002/11/15), Belgium (from 1991/01/02 to 2002/11/18), Brazil (from 1993/04/27 to 2004/09/20), Canada (from 1982/01/29 to 2002/11/15), Chile (from 1997/06/09 to 2002/10/14), Czech (from 1997/07/01 to 2004/09/20), Denmark (from 1989/12/04 to 2002/11/15), Egypt (from $1997 / 07 / 02$ to 2004/09/20), France (from 1987/07/09 to 2002/11/15), Germany (from 1959/10/01 to 2002/11/15), Hong Kong (from 1969/11/24 to 2004/09/21), India (from 1997/07/01 to 2004/09/20), Indonesia (from 1997/07/01 to 2004/09/20), Ireland (from 1987/03/09 to 2002/11/15), Israel (from 1992/10/08 to $2004 / 09 / 20$ ), Italy (from 1992/12/31 to 2002/11/15), Japan (from 1984/01/04 to $2004 / 09 / 17$ ), Korea (from $1997 / 07 / 01$ to $2004 / 09 / 20$ ), Malaysia (from $1977 / 01 / 03$ to $2002 / 11 / 15$ ), Mexico (from 1992/01/02 to $2002 / 11 / 15$ ), The Netherlands (from 1983/01/03 to 2002/11/15), New Zealand (from 1988/08/31 to 2002/11/15), Norway (from 1987/01/02 to 2002/11/15), Pakistan (from $1997 / 07 / 02$ to $2004 / 09 / 20$ ), Philippines (from 1997/07/02 to 2004/09/20), Russia (from 1997/07/01 to 2004/09/20), SP500 (from 1940/11/29 to 2004/09/21), Singapore (from 1985/01/04 to 2002/11/15), Slovakia (from 1997/07/01 to 2002/10/14), South Africa (from 1995/06/30 to 2002/11/15), Spain (from $1987 / 01 / 05$ to $2002 / 11 / 15$ ), Sri Lanka (from $1997 / 07 / 01$ to $2004 / 09 / 20$ ), Sweden (from 1986/12/18 to 2002/11/15), Switzerland (from 1989/07/03 to 2002/11/15), Taiwan (from 1997/07/02 to 2004/09/20), Thailand (from 1997/07/02 to 2004/09/20), Turkey (from 1997/07/01 to 2004/09/20), UK (from 1984/04/02 to 2004/09/21), Venezuela (from 2000/07/04 to 2002/09/05). We also observed a power-law distribution of the exit time with an exponent close to 1.5 .

As a typical example of emerging markets, we present in Fig. 2 the empirical probability distributions of the exit time of daily prices of the Shanghai Stock Exchange Composite, A Share Index, B Share Index, and 55 stocks listed in Shanghai Stock Exchange and Shenzhen Stock Exchange before 1992/12/01 at three different levels $\rho=0.00954,0.0596$, and 0.244 . One finds again that Eq. (4) holds and $\alpha \approx 1.5$.

So far, we have checked the validity of the new stylized fact in the daily evolutions of indexes and stock prices in both developed markets and emerging markets. To further clarify the situation, explorations were carried out on 10 high-frequency (5 minutes) data sets, including CAC 40 of France (covering July, August, September, and October of 2001, totally 9332 data points), S\&P 500 of USA (covering 1995, 1996, and 1997, totally 59885 data points), SSEC of China (from 2002/07/01 to 2004/09/16, totally 24955 data points), and seven stocks traded in the Shanghai Stock Exchange (six of them from June 2002, one from 2002/10/09, and one from 2003/07/25, all up to 2004/09/16). Again, 


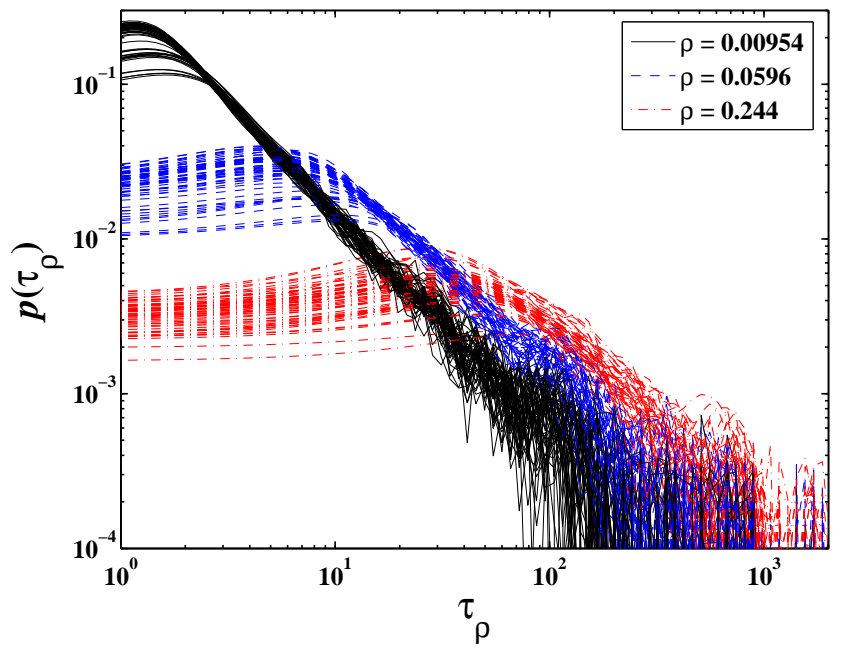

Fig. 2. The empirical probability distributions of the exit time of daily prices of the Shanghai Stock Exchange Composite, A Share Index, B Share Index, and 55 stocks listed in Shanghai Stock Exchange and Shenzhen Stock Exchange before 1992/12/01 at three different levels $\rho=0.00954,0.0596$, and 0.244 .

the scaling law (4) holds and $\alpha \approx 1.5$. Figure 3 illustrates the distributions of the data sets in the Chinese stock market.

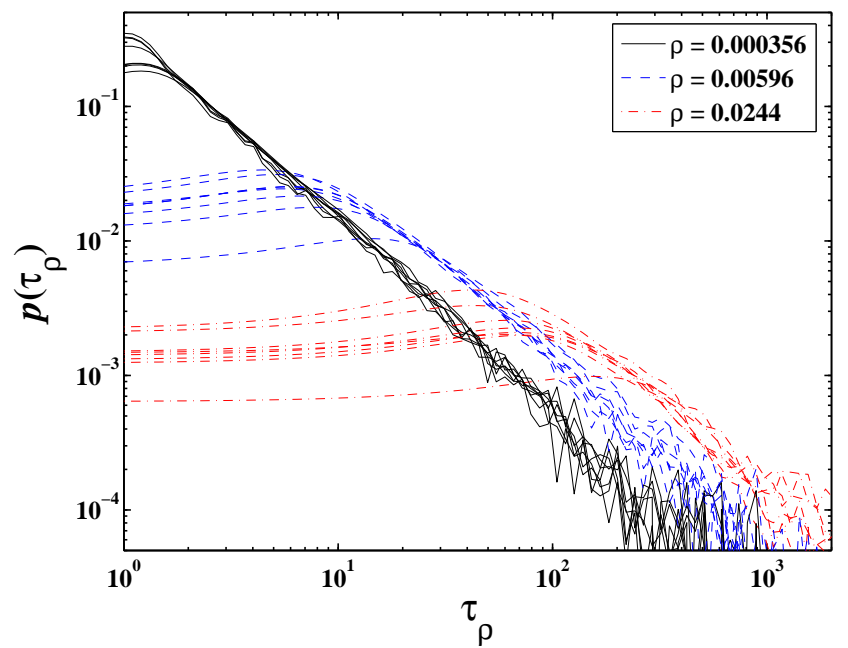

Fig. 3. The empirical probability distributions of the exit time of high-frequency (5 minutes) prices of the Shanghai Stock Exchange Composite and 7 stocks from 2002/07/01 (or 2002/10/09, or 2003/07/25) to 2004/09/16 in China at three different levels $\rho=0.000954,0.00596$, and 0.0244 .

We also analyzed the 1-minute-by-1-minute intraday NASDAQ data over 50 open days in the past from Monday Oct. 30, 2000. We observed a clear crossover from one power-law scaling to another separated with a kink around $\tau_{\rho} \approx 80$. The scaling exponent in the region $\tau_{\rho}^{*}<\tau_{\rho}<80$ is $\alpha_{1}=1.1$. The scaling exponent in the region $\tau_{\rho}>80$ is hard to estimate due to statistics. However, we can see that $\alpha_{2}$ of the second region is comparable to 1.5 or ever larger. This may signal a possible discrepancy between data sets of sampling 
frequency higher or lower than $1 / \mathrm{min}$. We shall come back to this in the future when longer data sets are available.

\section{Optimal investment horizon}

In the previous section, we verify the finding of a power-law scaling with an exponent of about $1.5[1,2,3]$ using numerous stock market indexes and individual stocks whose recording frequency is daily or $5 \mathrm{~min}$ all over the world. In this section, we focus on the power law relation (5) between the optimal investment horizon $\tau_{\rho}^{*}$ and the threshold $\rho$. We find that this power law (5) holds for all the data sets we studied in Section 2 but with quite different exponents.

For daily DJIA (1896-2004), we find that $\gamma \approx 1.55$, while S\&P 500 (1940-2004) and NASDAQ (1971-2002) give $\gamma \approx 1.5$. These values are significantly lower than those reported in $[1,2,3]$. This discrepancy may be due to the fact that we don't preprocess the data with a high-pass filter. Calculations with the 37 stocks in DJIA give similar results, as illustrated in Fig. 4.

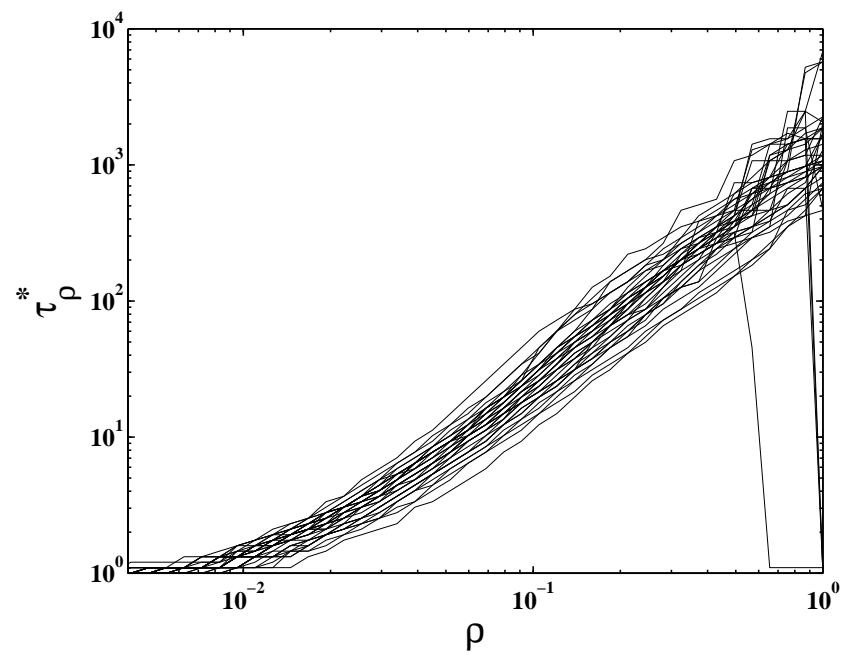

Fig. 4. The dependence of optimal investment horizon $\tau_{\rho}^{*}$ with respect to $\rho$ of daily prices of 37 stocks that are or were components of DJIA since 1962. The three lines with clear cutoffs at large $\rho$ correspond to stock prices with relatively short sizes.

For the 40 indexes in the stock markets throughout the world that we studied in the previous section, 22 stock markets have an exponent $\gamma \approx 1.50-1.55$. The rest 18 indexes have different $\gamma$ values. The Taiwan stock market index gives a higher scaling exponent $\gamma=1.64$, while the others have smaller $\gamma$ values (Belgium 1.40, Brazil 1.42, Chile 1.20, Czech 1.42, Egypt 1.20, Indonesia 1.35, Israel 1.40, Korea 1.39, Malaysia 1.36, New Zealand 1.40, Pakistan 1.37, Philippines 1.29, Russia 1.37, Singapore 1.38, Sri Lanka 1.39, Turkey 
1.33, Venezuela 1.0). With only a few exceptions, we see that most of the 22 stock markets having $\gamma \approx 1.50-1.55$ are developed markets, while most of 18 remaining indexes are emergent markets. In addition, Fig. 5 shows the dependence on $\rho$ of $\tau_{\rho}^{*}$ of 58 time series in the Chinese stock market. The value of $\gamma$ is around 1.25. Therefore, the exponent $\gamma$ is a kind of measure of the maturity of stock market in some sense.

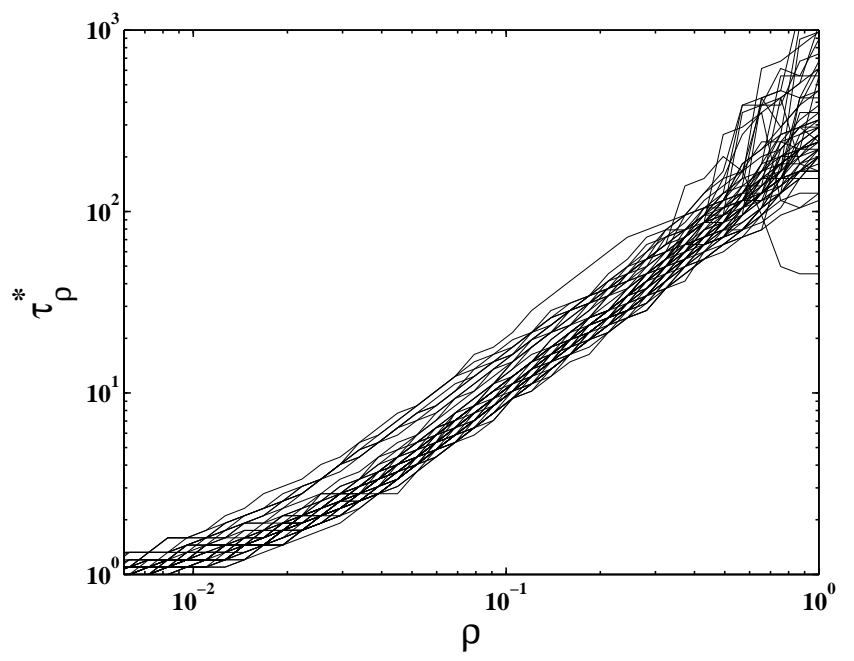

Fig. 5. The dependence of optimal investment horizon $\tau_{\rho}^{*}$ with respect to $\rho$ of daily prices of the Shanghai Stock Exchange Composite, A Share Index, B Share Index, and 55 stocks listed in Shanghai Stock Exchange and Shenzhen Stock Exchange before 1992/12/01.

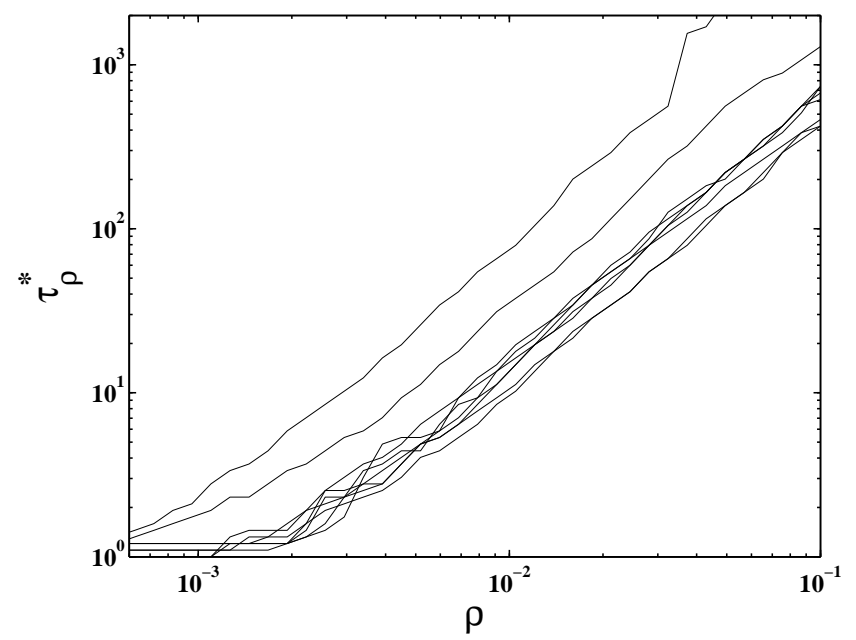

Fig. 6. The dependence of optimal investment horizon $\tau_{\rho}^{*}$ with respect to $\rho$ of high-frequency ( 5 minutes) prices of the Shanghai Stock Exchange Composite and 7 stocks from 2002/07/01 (or 2002/10/09, or 2003/07/25) in China.

In Fig. 6 shows the dependence of optimal investment horizon $\tau_{\rho}^{*}$ with respect to $\rho$ of high-frequency (5 minutes) prices of the Shanghai Stock Exchange Composite and 7 stocks in China. It is interesting to notice that the exponents of the six high-frequency time series in the Chinese stock markets are close 
to 1.50. The CAC 40 and S\&P 500 high-frequency data have $\gamma \approx 1.50$ as expected.

\section{Does the size matter?}

In Section 3, we have seen that the $\gamma$ values of most of the emerging markets are less than 1.4 and most of indexes or stock prices with $\gamma<1.4$ are from emerging markets. For an emerging market, the sizes of its recorded index and stock prices are usually short. In China, for instance, the first market for government-approved securities was founded in Shanghai on November 26, 1990 and started operating on December 19 of the same year under the name of the Shanghai Stock Exchange, and shortly after, the Shenzhen Stock Exchange was established on December 1, 1990 and started its operations on July 3, 1991 [24]. On the contrary, most of the major western stock markets have much longer histories. It is thus of great importance to check whether such a significant difference in scaling behaviors of the optimal investment horizon is due to the short size of the recorded time series in the emerging markets.

To understand this idiosyncratic scaling behavior in the emerging stock markets, we take the daily DJIA index from 1896 to 2004 as a proxy. This time series has 29493 data points. We run a moving window of size 3500 (about 14 years) along the evolution of DJIA with a step of 20 trading days. This gives 1300 moving windows. For each window, we determine its exit times of all points separately. In other words, each running window is treated as an independent time series. Then the optimal in vestment horizon $\tau_{\rho}^{*}$ is determined for each window at 21 threshold levels logarithmically spaced in [0.0194, 0.3237]. To estimate the value of $\gamma$ for each window, we require a linear regression coefficient larger than 0.995 . If this constraint is not fulfilled, we remove the last point and regress the remaining data again. This iterative procedure repeats again and again until the linear regression coefficient is larger than 0.995. For most of the data sets, this constraint satisfies with all the 21 points.

The probability distribution $p(\gamma)$ of the estimated $\gamma$ 's is plotted in Fig. 7 with solid line. The maximum locates at $\gamma_{\max }=1.51$. The percentage of the windows with $\gamma<1.4$ is $13.85 \%$. When the Chinese stock markets are concerned, only 22 windows have $\gamma<1.3$, implying a percentage of $1.69 \%$. This result already supports the point that there is little chance that the idiosyncratic scaling behavior in the Chinese stock markets could not be attributed to its short history and this property is thus of little probability to be artificial. This conclusion is still significant although less strong in other emergent markets.

To further enhance our point, we have taken a closer look at the 180 regressions 


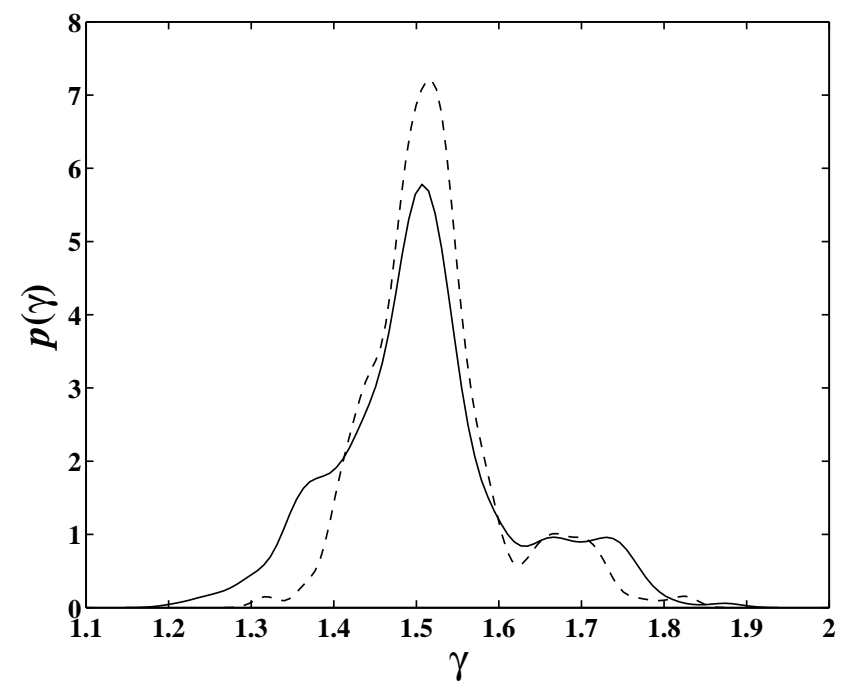

Fig. 7. Probability distribution of the estimated scaling exponent $\gamma$. The probability distributions are estimated with Gaussian kernel smoothing approach.

where $\gamma<1.4$. Figure 8 shows the dependence of $\tau_{\rho}^{*}$ against $\rho$ for all the 180 windows. There are three clusters in Fig. 8. The lines in the bottom cluster exhibit a sudden jump around $\rho=0.08$. The left parts with relatively small $\rho$ of all lines are linear with a slope of 1.42 . The right parts show slightly downward bending. If we fit the points in the left part for each line, we find that the percentage of running windows with $\gamma<1.4$ reduces dramatically to $2.15 \%$, while the number of windows with $\gamma<1.3$ is null.

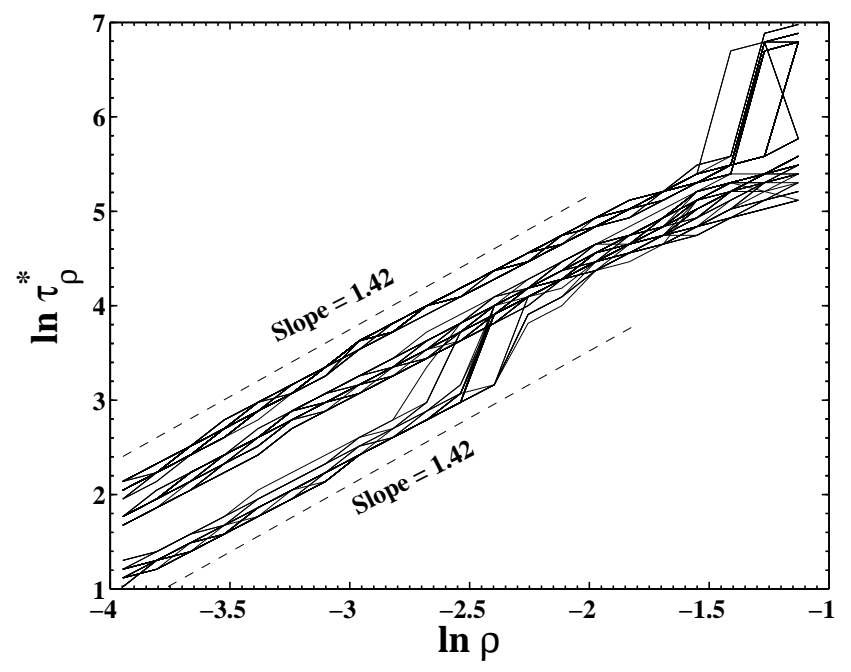

Fig. 8. Dependence of $\tau_{\rho}^{*}$ against $\rho$ for the 180 windows where $\gamma<1.4$.

In Fig. 7, one observes that there are also quite a few windows whose $\gamma>1.6$. The number of these windows is 214 , a percentage of $16.46 \%$. Figure 9 shows the dependence of $\tau_{\rho}^{*}$ against $\rho$ for all the 214 windows. For large $\rho$, the linearity deteriorates remarkably. The slopes of these lines are comparable to 1.65. If we redo the regressions after excluding the points at large $\rho$, the percentage of 
windows with $\gamma>1.6$ reduces to $12.31 \%$. The probability distribution $p(\gamma)$ of the modified $\gamma$ 's is plotted in Fig. 7 with dashed line. The maximum locates at $\gamma_{\max }=1.52$. The kurtosis excess of the distribution in dashed line is 4.56, higher than that in solid line with a value of 3.50 .

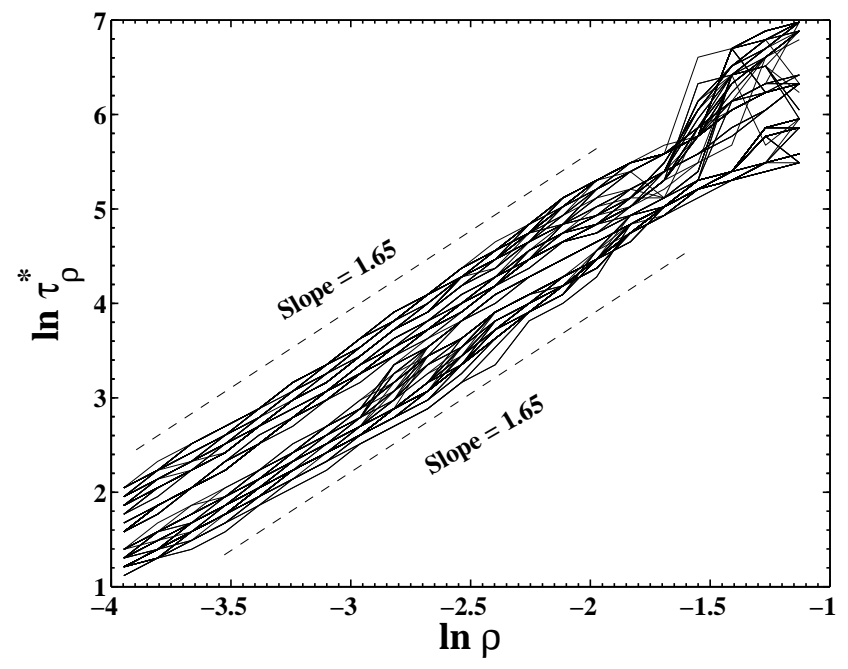

Fig. 9. Dependence of $\tau_{\rho}^{*}$ against $\rho$ for the 180 windows where $\gamma>1.6$.

At last, let us average $\tau_{\rho}^{*}$ over the 1300 running windows for each threshold $\rho$. A perfect power law is observed as follows

$$
\ln \left\langle\tau_{\rho}^{*}\right\rangle=1.56 \ln \rho+7.86
$$

The linear regression coefficient is 0.9997 . The power-law exponent 1.56 is slightly larger than the $\gamma_{\max }$ due to the fact that the skewness of the two distributions shown in Fig. 7 are 0.46 and 0.96, respectively.

\section{Concluding remarks}

There are universal and idiosyncratic behaviors in the developed and emerging stock markets, such as the log-periodic power-law antibubble pattern [25] or return anomalies [26] in China's stock markets. In this paper, we have verified that the probability density of exit time follows a universal power law with exponent $\alpha=1.5$ for large $\tau_{\rho}$ for daily indexes and stock prices and 5-min high-frequency data in both the developed and emerging markets. However, this statistical property can not be extrapolated to other financial markets, at least not to the tick-by-tick data in the foreign exchange markets where the power law exponent $\alpha \approx 2.4$ is remarkably greater than the one in stock markets [11].

On the other hand, although the optimal investment horizon $\tau_{\rho}^{*}$ scales as 
$\rho^{\gamma}$ for all stock markets investigated, they have quite different values of $\gamma$. Roughly speaking, the $\gamma$ values of the daily data in emerging stock markets are significantly less than those in major western markets $(\gamma \approx 1.5)$. We have showed that this discrepancy in $\gamma$ couldn't stems from the difference of record sizes in the two kinds of stock markets. In words, for a given gain threshold, the optimal investment horizon in an emerging market is shorter than that in a developed market. This is consistent with the known result that the optimal trading time lag is about 30 days in China's stock markets, much shorter than that in the USA markets, due to the speculative nature of emerging markets $[25]$.

For 5-min high-frequency data (CAC 40 index, S\&P 500 index, and SSEC and stock prices in China) we find that $\gamma \approx 1.5$. This intriguing feature can be interpreted by the $t+1$ trading rule in the Chinese stock markets which reduces the speculation at high-frequency levels. As a cautionary note, we notice that the 1-min high-frequency data of NASDAQ do not follow the simple power-law relation in the exit time distribution, which calls for a further investigation.

\section{Acknowledgments:}

We are grateful to Wei Deng for providing us the numerous data sets of the indexes and individual stock prices in the Chinese stock markets and thank Didier Sornette for careful reading of the manuscript. This work was jointly supported by NSFC/PetroChina through a major project on multiscale methodology.

\section{References}

[1] I. Simonsen, M. H. Jensen, A. Johansen, Optimal investment horizons, Eur. Phys. J. B 27 (2002) 583-586.

[2] M. H. Jensen, A. Johansen, I. Simonsen, Inverse statistics in economics: the gain-loss asymmetry, Physica A 324 (2003) 338-343.

[3] M. H. Jensen, A. Johansen, I. Simonsen, Inverse fractal statistics in turbulence and finance, Int. J. Mod. Phys. B 17 (2003) 4003-4012.

[4] B. B. Mandelbrot, The variation of certain speculative prices, J. Business 36 (1963) 394-419.

[5] R. N. Mantegna, H. E. Stanley, An Introduction to Econophysics: Correlations and Complexity in Finance, Cambridge University Press, Cambridge, 2000.

[6] J.-P. Bouchaud, M. Potters, Theory of Financial Risks: From Statistical Physics to Risk Management, Cambridge University Press, Cambridge, 2000. 
[7] D. Sornette, Why Stock Market Crashes: Critical Events in Complex Financial Systems, Princeton University Press, Princeton, 2003.

[8] R. Cont, Empirical properties of asset returns: stylized facts and statistical issues, Quantitative Finance 1 (2001) 223-236.

[9] S. Ghashghaie, W. Breymann, J. Peinke, P. Talkner, Y. Dodge, Turbulent cascades in foreign exchange markets, Nature 381 (1996) 767-770.

[10] R. N. Mantegna, H. E. Stanley, Turbulence and financial markets, Nature 383 (1996) 587-588.

[11] M. H. Jensen, A. Johansen, F. Petroni, I. Simonsen, Inverse statistics in the foreign exchange market, Physica A 340 (2004) 678-684.

[12] M. H. Jensen, Multiscaling and structure functions in turbulence: An alternative approach, Phys. Rev. Lett. 83 (1999) 76-79.

[13] L. Biferal, M. Cencini, D. Vergni, A. Vulpiani, Exit time of turbulent signals: A way to detect the intermediate dissipative range, Phys. Rev. E 60 (1999) R6295-R6298.

[14] M. Abel, L. Biferal, M. Cencini, M. Falcioni, D. Vergni, A. Vulpiani, Exit-times and $\epsilon$-entropy for dynamical systems, stochastic processes, and turbulence, Physica D 147 (2000) 12-35.

[15] L. Biferal, M. Cencini, A. S. Lanotte, D. Vergni, A. Vulpiani, Inverse statistics of smooth signals: The case of two dimensional turbulence, Phys. Rev. Lett. 87 (2001) 124501.

[16] L. Biferal, M. Cencini, A. S. Lanotte, D. Vergni, Inverse velocity statistics in two-dimensional turbulence, Phys. Fluids 15 (2003) 1012-1020.

[17] S. Beaulac, L. Mydlarski, Inverse structure functions of temperature in gridgenerated turbulence, Phys. Fluids 16 (2004) 2126-2129.

[18] S. Roux, M. H. Jensen, Dual multifractal spectra, Phys. Rev. E 69 (2004) 016309.

[19] M.-Z. Ding, W.-M. Yang, Distribution of the first return time in fractional brownian motion and its application to the study of on-off intermittency, Phys. Rev. E 52 (1995) 207-213.

[20] G. Rangarajan, M.-Z. Ding, Anomalous diffusion and the first passage time problem, Phys. Rev. E 61 (2000) 120-133.

[21] G. Rangarajan, M.-Z. Ding, First passage time distribution for anomalous diffusion, Phys. Lett. A 273 (2000) 322-330.

[22] G. Rangarajan, M.-Z. Ding, First passage time problem for biased continuoustime random walks, Fractals 8 (2000) 139-145.

[23] A. Bowman, A. Azzalini, Applied Smoothing Techniques for Data Analysis, Oxford University Press, Oxford, 1997. 
[24] D.-W. Su, Chinese Stock Markets: A Research Handbook, World Scientific, Singapore, 2003.

[25] W.-X. Zhou, D. Sornette, Antibubble and prediction of china's stock market and real-estate, Physica A 337 (2004) 243-268.

[26] R. Mookerjee, Q. Yu, An empirical analysis of the equity markets in china, Review of Financial Economics 8 (1999) 41-60. 